\title{
Large Strain Field Near a Crack Tip in a Rubber Sheet
}

\author{
Y C Gao $\quad$ S H Chen"* \\ * Northem Jiaotong University, 100044, Beijıng, China
}

** LNM, Institute of Mechanics, Chinese Academy of Sciences, 100080, Beııng, China (Recelved 10 June 2000, accepted for print 1 November 2000)

\section{Abstract}

The distribution of stress-strain near a crack tip in a rubber sheet is investigated by employing the constitutive relation given by Gao (1997) It is shown that the crack tip field is composed of two shrinking sectors and one expanding sector The stress state near the crack tip is in uniaxial tension The analytical solutions are obtained for both expanding and shrinking sectors

Key words. large strain, rubber sheet, crack

\section{Introduction}

The large strain elastic field near a crack tip is a difficult but very typical problem that can examine various elastic laws and analytical methods The feature of the field depends on the elastic law Knowles and Sternberg (1973) proposed an elastic law for rubber materials, and analyzed the mode I crack tip field under plane strain condition Gao $(1990,1997)$ proposed two elastic laws and gave a sector division method, those are used to analyze the mode I plane strain crack tip field The interesting fact is that according to the three different elastic laws mentioned above, the very similar stress fields near a crack tip are obtained, but Knowles and Sternberg $(1973,1974)$ did not consider the expanding sector Gao and Gao (1999) analyzed the large strain notch tip (crack is a special case) field and compared the three elastic laws It is found that for notch tip field, the three elastic laws can become equivalent if the constnutive parameters tre related by some conditions It is also found that when the expanding sector is taken into account the restriction on parameter $n$ (given by Knowles and Sternberg) is not needed

Comparing with plane strain problem, the plane stress crack tip field is more complicated because of the thickness shrinkage An asymptotic analysis of the plane stress crack was given by Gao and Durban (1995) where the thickness shrinkage can be expressed by strain invariants For the elastıc law given by Gao (1997), the thickness shrınkage can only be given by a differential equation that must be solved numerically The purpose of this paper is to reveal the crack tip feature in a sheet that obeys the elastic law given by Gao (1997)

\section{Basic Equations}

Consider a three-dimensional elastic body Let $\mathbf{P}$ and $\mathbf{Q}$ denote the position vectors of a point before and after deformation, respectively $x^{l}(t=1,2,3)$ denote Lagrangıan coordinates Two sets of triads are defined as

$$
\mathbf{P}_{1}=\frac{\partial \mathbf{P}}{\partial x^{\prime}} \quad \mathbf{Q}_{1}=\frac{\partial \mathbf{Q}}{\partial x^{\prime}}
$$

The displacement gradient is

$$
\mathbf{F}=\mathbf{Q}, \otimes \mathbf{P}^{\prime}
$$

Note that $\boldsymbol{P}^{\prime}$ is the conjugate of $\boldsymbol{P}_{1}$, while $\otimes$ is the dyadic symbol and summation rule is implied

The Green and Cauchy deformation tensors are

$$
D=F^{T} F \quad d=F F^{T}
$$

\footnotetext{
*Email ycgao@center njtu edu cn
} 
where the superscript $T$ denotes the transpose Let $\mathbf{E}$ stand for the unit tensor, then the following invariants will be used,

$$
I_{1}=D \quad E=d \quad E, I_{-1}=D^{-1} \quad E=d^{-1} \quad E, J=V_{Q} / V_{P}
$$

where $V_{Q}=\left(Q_{1}, Q_{2}, Q_{3}\right), V_{P}=\left(P_{1}, P_{2}, P_{3}\right)$, and the brackets denote the mixed product

A kind of strain energy per undeformed unit volume was proposed in Gao (1997),

$$
U=a\left(I_{1}^{n}+I_{-1}^{n}\right)
$$

where $a$ and $n$ are material constants Then the Kirchhoff stress is

$$
a=2 \frac{\partial U}{\partial D}=2 n a\left(I^{n-1} E-I_{-1}^{n-1} D^{-2}\right)
$$

From $\mathrm{Eq}(6)$, the Cauchy stress can be obtained

$$
\tau=J^{-1} F \quad \sigma F^{T}=2 n a J^{-1}\left(I_{1}^{n-1} d-I_{-1}^{n-1} d^{-1}\right)
$$

The equilibrium equation is

$$
\frac{\partial\left(V_{Q} \tau Q^{\prime}\right)}{\partial x_{1}}=0
$$

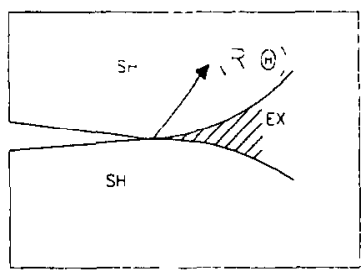

(a)

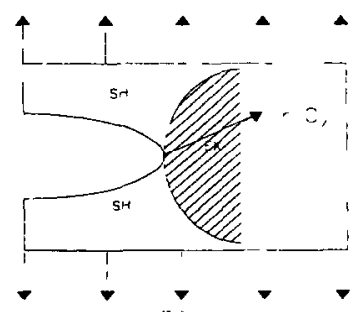

(b)

Fig 1 The sector division near the crack tıp, (a) before loadıng, (b) after loading

\section{Shrinking Sector SH}

Fig 1 (a) and (b) show the cracked rubber sheet before and after loading respectively Since the strain near the crack tip is very large, as analyzed in Gao (1990), the deformation cannot be described by an uniformed mapping function for the whole region According to the sector division method, the whole crack tip field is divided into one expanding sector EX and two shrinkıng sectors SH and SH' Before loading EX is very narrow while SH and SH' occupy almost the whole crack tip field After loading EX occupies the whole crack tip field while SH and SH' become very narrow Two Lagrangian coordinates are introduced, $(R, \Theta, Z)$ is the cylindrical coordinate before deformation and $(r, \theta, z)$ is the cylindrical coordinate after loading

For the shrinking sectors $\mathrm{SH}$, the mapping function from $(R, \Theta, Z)$ to $(r, \theta, z)$ is made as follows,

$$
r=R^{1-\delta} \varphi(\Theta), \quad \theta=\frac{\pi}{2}-R^{r} \psi(\Theta), \quad z=R^{\prime} Z \eta(\Theta)
$$

in which $\delta, \gamma, t>0,0<\Theta \leq \pi \quad t>0$ indicates that the thickness of the sheet shrinks tremendously From Eq (9), the local triads are

$$
\left\{\begin{array}{l}
Q_{R}=R^{-\delta} \varphi\left[(1-\delta) e_{r}-\gamma R^{\gamma} \psi e_{\theta}\right]+R^{t-1} t Z \eta e_{z} \\
Q_{\Theta}=R^{1-\delta}\left(\varphi^{\prime} e_{r}-R^{\gamma} \varphi \psi^{\prime} e_{\theta}\right)+R^{t} Z \eta^{\prime} e_{z} \\
Q_{Z}=R^{t} \eta e_{z}
\end{array}\right.
$$

where

$$
e_{r}=Q_{r}=\frac{\partial Q}{\partial}, \quad e_{\theta}=\frac{1}{r} Q_{\theta}=\frac{1}{r} \frac{\partial Q}{\partial \theta}, \quad e_{z}=Q_{z}=\frac{\partial Q}{\partial z}
$$


It is assumed that the thickness of the sheet is much smaller than the size of considered domain, therefore the terms with $Z$ in Eq (10) can be neglected

Substituting Eqs (10) and (2) into (3) and noting that

$$
\begin{array}{lll}
P^{R} P^{R}=1, & P^{\Theta} P^{\Theta}=R^{-2}, \\
P^{Z} & P^{Z}=1, & P^{\prime} P^{J}=0, \quad(i, J=R, \Theta, Z \text { and } \quad l \neq j)
\end{array}
$$

we obtain the dominant terms of $d$ and $d^{-1}$,

$$
\left\{\begin{aligned}
d= & R^{-2 \delta}\left[T e_{r} e_{r}-R^{\gamma} S\left(e_{r} e_{\theta}+e_{\theta} e_{r}\right)+R^{2 \gamma} V e_{\theta} e_{\theta}\right]+R^{2 t} \eta^{2} e_{z} e_{z} \\
d^{-1}= & R^{2 \delta-2 \gamma} q^{-2}\left[T e_{\theta} e_{\theta}+R^{\gamma} S\left(e_{r} e_{\theta}+e_{\theta} e_{r}\right)+R^{2 \gamma} V e_{r} e_{r}\right] \\
& +R^{-2 t} \eta^{-2} e_{z} e_{z}
\end{aligned}\right.
$$

in which

$$
\begin{cases}q=\varphi\left[\gamma \varphi^{\prime} \psi-(1-\delta) \varphi \psi^{\prime}\right] & T=\varphi^{\prime 2}+(1-\delta)^{2} \varphi^{2} \\ S=\varphi\left[\gamma(1-\delta) \varphi \psi+\varphi^{\prime} \psi^{\prime}\right] & V=\varphi^{2}\left(\gamma^{2} \psi^{2}+\psi^{\prime 2}\right)\end{cases}
$$

The strain invariants are

$$
\left\{\begin{array}{l}
I_{1}=R^{-2 \delta} T, \\
J=R^{\gamma+t-2 \delta} \eta q
\end{array} \quad I_{-1}=R^{2 \delta-2 \gamma} q^{-2} T+R^{-2 t} \eta^{-2}\right.
$$

in the coordinate $R, \Theta, Z$, we have

$$
V_{Q}=R^{1+\gamma+t-2 \delta} \eta q
$$

We assume that the shrinkage in the thickness direction and in $\Theta$ direction is the same order, then the two terms in the expression of $I_{-1}$ must be the same order, so

$$
t=\gamma-\delta
$$

For the plane stress problem, since $\tau_{z z}=0$, according to Eqs (13)-(15), (17) and (7) we obtain

$$
t=\frac{n-1}{n+1} \delta, \quad \gamma=\frac{2 n}{n+1} \delta
$$

and

$$
T^{n-1} \eta^{4}=\left(q^{-2} T+\eta^{-2}\right)^{n-1}
$$

then

where $\quad \lambda=2 n \delta+2 \gamma-3 \delta$

$$
\begin{aligned}
\tau= & 2 n a \eta^{-1} q^{-1} T^{n-1} R^{-\lambda}\left[T e_{r} \otimes e_{r}-R^{\gamma} S\left(e_{r} \otimes e_{\theta}+e_{\theta} \otimes e_{r}\right)\right. \\
& \left.+R^{2 \gamma}\left(V-T \eta^{4} q^{-2}\right) e_{\theta} \otimes e_{\theta}\right]
\end{aligned}
$$

Eq (20) indicates that the dominant component of stress is $\tau_{r r}$

Substıtutıng (20) and (16) into (8), noting that

$$
\begin{cases}\frac{\partial e_{r}}{\partial R}=-\gamma R^{\gamma-1} \psi e_{\theta}, & \frac{\partial e_{r}}{\partial \Theta}=-R^{\gamma} \psi^{\prime} e_{\theta} \\ \frac{\partial e_{\theta}}{\partial R}=\gamma R^{\gamma-1} \psi e_{r}, & \frac{\partial e_{\theta}}{\partial \Theta}=R^{\gamma} \psi^{\prime} e_{r}\end{cases}
$$

and

$$
\begin{aligned}
& \left\{\begin{array}{l}
Q^{R}=-R^{\delta-\gamma} q^{-1}\left(R^{\gamma} \varphi \psi^{\prime} e_{r}+\varphi^{\prime} e_{\theta}\right) \\
Q^{\Theta}=R^{\delta-1-\gamma} q^{-1} \varphi\left[R^{\gamma} \gamma \psi e_{r}+(1-\delta) e_{\theta}\right]
\end{array}\right. \\
& \begin{cases}\varphi \psi^{\prime} T-u \varphi^{\prime}=-(1-\delta) \varphi q, & T \gamma \psi-(1-\delta) S=\varphi^{\prime} q / \varphi \\
-S \gamma \psi+(1-\delta) V=-q \psi^{\prime}, & -\varphi \psi^{\prime} S+\varphi^{\prime} V=\gamma \varphi \psi q\end{cases}
\end{aligned}
$$

After extensive mantpulation of these equations we obtain

$$
\left[1+\frac{2(n-1)}{T} \varphi^{\prime 2}\right]\left[\varphi^{\prime \prime}+(1-\delta)^{2} \varphi\right]-(1-\delta)(1-2 \delta) \varphi=0
$$

and 


$$
\begin{aligned}
& {\left[\varphi+3(1-\delta)^{2} T \varphi^{3} \eta^{4} q^{-4}\right] \psi^{\prime \prime}+\left[2(n-1) \varphi \varphi^{\prime} \psi^{-1}\right.} \\
& \left.+2 n(1-\delta) \varphi \varphi^{\prime} \eta^{4} q^{-3}-3(1-\delta) \gamma T \varphi^{2} \psi \eta^{4} q^{-4}\right] \varphi^{\prime \prime} \\
& +2(1-\delta)^{2} \varphi \varphi^{\prime}\left[(n-1) \varphi \psi^{\prime} T^{-1}+(1-\delta) n \varphi \eta^{4} q^{-3}\right] \\
& +\gamma(1+\gamma) \varphi \psi-(2 \delta+\gamma-1) q^{-3} T \eta^{4} \varphi^{\prime}+2 \varphi^{\prime} \psi^{\prime} \\
& +4(1-\delta) T \varphi \eta^{3} q^{-3} \eta^{\prime} \\
& -3(1-\delta) T \varphi \eta^{4} q^{-4}\left[\gamma \varphi^{\prime 2} \psi-(2-2 \delta-\gamma) \varphi \varphi^{\prime} \psi^{\prime}\right]=0
\end{aligned}
$$

in which

$$
\begin{aligned}
& (n-1)\left(q^{-2} T^{\prime} \eta^{-2}\right)^{n-2}\left[-2 q^{-3} q^{\prime} T+q^{-2} T^{\prime}-2 \eta^{-3} \eta^{\prime}\right] \\
& -(n-1) T^{n-2} T^{\prime} \eta^{4}-4 T^{n-1} \eta^{3} \eta^{\prime}=0
\end{aligned}
$$

$$
\left\{\begin{array}{l}
q^{\prime}=\gamma \varphi \varphi^{\prime \prime} \psi-(1-\delta) \varphi^{2} \psi^{\prime \prime}+\gamma \varphi^{\prime 2} \psi-(2-2 \delta-\gamma) \varphi \varphi^{\prime} \psi^{\prime} \\
T^{\prime}=2 \varphi^{\prime} \varphi^{\prime \prime}+2(1-\delta)^{2} \varphi \varphi^{\prime}
\end{array}\right.
$$

In order to match the displacements in sectors SH and EX, Eqs (25)-(27) should meet the natural boundary conditions

$$
\varphi(0)=0, \quad \psi \quad \psi(0)=\infty
$$

At $\Theta=\pi$, the traction free conditions can be reduced to

$$
\varphi^{\prime}(\pi)=0 \quad \psi^{\prime}(\pi)=\frac{-\eta(\pi)}{\varphi(\pi)}
$$

Eq (19) gives,

$$
\eta(\pi)=\left[\frac{(1-\delta)^{2} \varphi_{(\pi)}^{2}}{2}\right]^{\frac{1-n}{2(1+n)}}
$$

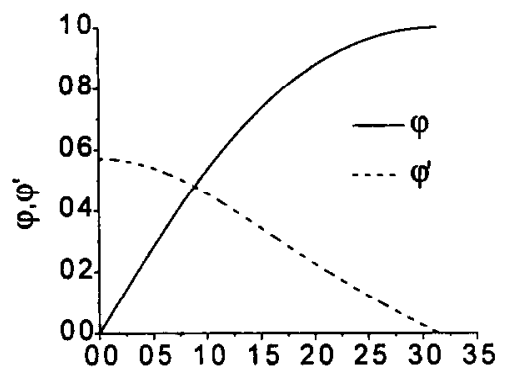

$\Theta$

Fig 2 The curves of $\varphi(\Theta)$ and $\varphi^{\prime}(\Theta)$

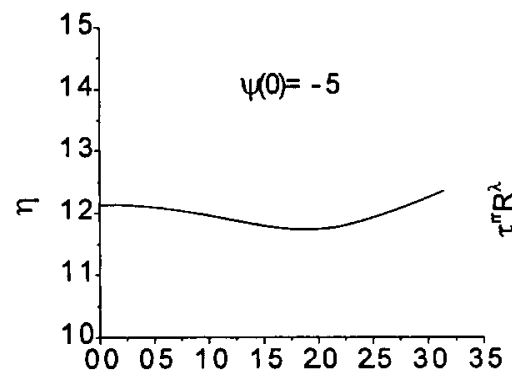

$\Theta$

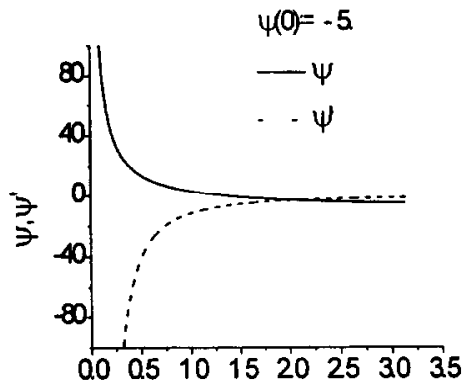

$\Theta$

Fig 3 The curves of $\psi(\Theta)$ and $\psi^{\prime}(\Theta)$

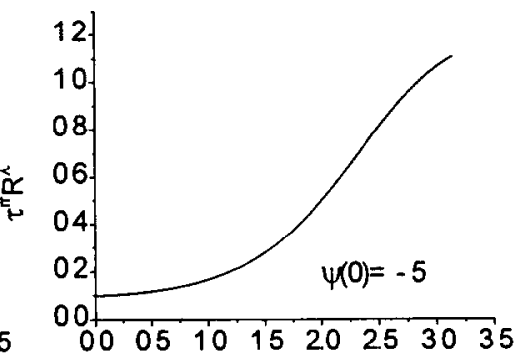

$\Theta$

Fig 4 The curve of $\eta(\Theta)$

Fig 5 The curves of normalized stress $\tau^{r r} R^{\lambda}$ 
The detaled solution of Eq (25) subjected to the conditions (29) and (30) was given in Gao and Gao (1999) The eigenvalue $\delta$ is

$$
\delta=\frac{1}{2 n}
$$

The analytical solution of $\varphi$ is

$$
\varphi=\frac{\sqrt{2}}{2} \varphi_{\pi} n^{\frac{1}{2}-\frac{1}{2 n}}(\Omega-\cos \theta)^{1 / 2}\left[\Omega+\left(1-\frac{1}{n}\right) \cos \theta\right]^{\frac{1}{2}-\frac{1}{2 n}}
$$

where

$$
\Omega=\left[1-\left(1-\frac{1}{n}\right)^{2} \sin ^{2} \Theta\right]^{1 / 2}
$$

where $\varphi_{\pi}=\varphi(\pi)$ is a parameter to indicate the amplitude of the field The value of $\varphi_{\pi}$ depends on the load at far field When $\varphi_{\pi}$ is given, the function $\psi$ and $\eta$ can be solved numerically from Eqs (26)-(30) The functions $\varphi, \varphi^{\prime}, \psi, \psi^{\prime}, \eta, \tau_{r r} R^{\lambda}$ are shown in Fig $2 \sim 5$ for the case $n=20$, $\varphi(\pi)=1, \psi(\pi)=-50$

Eq (32) is consistent with an analysis based on energy considerations Actually, at the crack tip, the energy density must be of the order $R^{-1}$, then from Eqs (5), (15) we have

$$
2 n \delta=1
$$

\section{Expanding Sector}

The deformation pattern (9) is not valid when $\Theta \rightarrow 0$ because $\varphi \rightarrow 0$ and $\psi \rightarrow \infty$ Therefore, the problem must be considered in EX sector $\left(\Theta \sim R^{\alpha}\right)$, where the mapping functions are assumed to be

$$
\begin{cases}r=R^{1+\beta} \rho(\xi), & \theta=\frac{\pi}{2}-\omega(\xi) \\ z=R^{b} Z \zeta(\xi), & \xi=\Theta R^{-\alpha}\end{cases}
$$

where $\alpha, \beta, b$ are positive constants to be determined From Eq (1) and (36), we obtain

$$
\left\{\begin{array}{l}
\boldsymbol{Q}_{R}=R^{\beta}\left\{\left[(1+\beta) \rho-\alpha \xi \rho^{\prime}\right] e_{r}+\alpha \xi \rho \omega^{\prime} e_{\theta}\right\}+R^{b-1}\left(b \varsigma-\alpha \xi \varsigma^{\prime}\right) Z e_{z} \\
\boldsymbol{Q}_{\Theta}=R^{\beta-\alpha+1}\left(\rho^{\prime} e_{r}-\rho \omega^{\prime} e_{\theta}\right)+R^{b-\alpha} Z \varsigma^{\prime} e_{z} \\
\boldsymbol{Q}_{Z}=R^{b} \varsigma e_{z}
\end{array}\right.
$$

Since the thickness of the sheet is assumed to be small, the terms with $Z$ in Eq (37) can be neglected

Combining Eqs (37), (2), (3), (12), we can obtain the dominant terms of $d$ and $d^{-1}$,

$$
\begin{gathered}
d=R^{-2 \alpha+2 \beta}\left[\rho^{\prime 2} e_{r} e_{r}+\rho^{2} \omega^{\prime 2} e_{\theta} e_{\theta}\right. \\
\left.-\rho \rho^{\prime} \omega^{\prime}\left(e_{r} e_{\theta}+e_{\theta} e_{r}\right)\right]+R^{2 b} \varsigma^{2} e_{z} e_{z} \\
d^{-1}=R^{-2 \beta} v^{-2}\left[\rho^{2} \omega^{\prime 2} e_{r} e_{r}+\rho^{\prime 2} e_{\theta} e_{\theta}+\right. \\
\left.\rho \rho^{\prime} \omega^{\prime}\left(e_{r} e_{\theta}+e_{\theta} e_{r}\right)\right]+R^{-2 b} \varsigma^{2} e_{z} e_{z}
\end{gathered}
$$

in which

$$
v=-(1+\beta) \rho^{2} \omega^{\prime}
$$

The invariants are

where

$$
I_{1}=R^{2 \beta-2 \alpha} u, \quad I_{-1}=R^{-2 \beta} u v^{-2}+R^{-2 b} \varsigma^{-2}, J=R^{2 \beta+b-\alpha} \varsigma \nu
$$

$$
u=\rho^{\prime 2}+\rho^{2} \omega^{\prime 2}
$$

In the coordinate $(R, \Theta, Z)$

$$
V_{Q}=R^{2 \beta+b-\alpha+1} \mathcal{S}
$$

Assuming the shrinkage along the thickness direction and $\Theta=0$ direction are the same order 
then the two terms in $I_{-1}$ should be the same order, so

$$
b=\beta
$$

Substıtutıng Eqs (38)-(41) into (7) and notıng (44) and that $\tau_{z z}=0$, it follows

$$
\begin{aligned}
& \alpha=\frac{2 n}{n-1} \beta \\
& u^{n-1} \varsigma^{2}-\left(u v^{-2}+\varsigma^{-2}\right)^{n-1} \varsigma^{-2}=0
\end{aligned}
$$

Noting the following relations

$$
\begin{aligned}
& \frac{\partial \xi}{\partial \Theta}=R^{-\alpha}, \quad \frac{\partial R}{\partial \Theta}=0, \quad \frac{\partial \theta}{\partial \Theta}=-\omega^{\prime} R^{-\alpha} \\
& \frac{\partial e_{r}}{\partial \Theta}=-R^{-\alpha} \omega^{\prime} e_{\theta}, \quad \frac{\partial e_{\theta}}{\partial \Theta}=R^{-\alpha} \omega^{\prime} e_{r} \\
& \left\{\begin{array}{l}
Q^{R}=-R^{-\beta} v^{-1}\left(\rho \omega^{\prime} e_{r}+\rho e_{\theta}\right) \\
Q^{\Theta}=R^{\alpha-1-\beta} v^{-1}\left\{\alpha \xi \rho \omega^{\prime} e_{r}-\left[(1+\beta) \rho-\alpha \xi \rho^{\prime}\right] e_{\theta}\right\}
\end{array}\right.
\end{aligned}
$$

then $\quad \tau Q^{R} \ll \tau Q^{\Theta}$

Eq (8) can be reduced to

$$
\frac{\partial\left(V_{Q} \tau Q^{\Theta}\right)}{\partial \Theta}=0
$$

finally, it is obtained that,

$$
\left\{\begin{array}{l}
\rho \omega^{\prime \prime}+2 \rho^{\prime} \omega^{\prime}=0 \\
\rho^{\prime \prime}-\rho \omega^{\prime 2}=0
\end{array}\right.
$$

The boundary conditions for (52) at $\xi=0$ are

$$
\begin{array}{ll}
\rho^{\prime}(0)=0, & \rho(0)=\rho_{0} \\
\omega(0)=\frac{\pi}{2}, & \left(^{\prime}(0)=-r\right.
\end{array}
$$

$\rho_{0}$ and $c$ are constants to be determined The solution of (52) is

$$
\rho=\rho_{0}\left(c^{2} \xi^{2}+1\right)^{1 / 2}, \quad \omega=\frac{\pi}{2}-\operatorname{arctg}(c \xi)
$$

It is easy to prove that

$$
\begin{array}{ll}
\rho^{\prime}=\frac{\rho_{0}^{2}}{\rho}\left(c^{2} \xi^{2}+1\right)^{1 / 2}, & \omega^{\prime}=-\frac{c}{\rho^{2}} \rho_{0}^{2} \\
u=c^{2} \rho_{0}^{2}, & v=(1+\beta) c \rho_{0}^{2}
\end{array}
$$

then according to (7) and (38) we have

$$
\begin{aligned}
& \tau \sim \rho^{\prime 2} e_{r} e_{r}+\rho^{2} \omega^{\prime 2} e_{\theta} e_{\theta}-\rho \rho^{\prime} \omega^{\prime}\left(e_{r} e_{\theta}+e_{\theta} e_{r}\right) \\
& =c^{2} \rho_{0}^{2}\left[\sin ^{2} \theta e_{r} e_{r}+\cos ^{2} \theta e_{\theta} e_{\theta}+\sin \theta \cos \theta\left(e_{r} e_{\theta}+e_{\theta} e_{r}\right)\right]
\end{aligned}
$$

From Eq (58) we conclude that the expanding sector is in uniaxial tension state Now we analyze the parameter $\rho_{0}$ and $c$ From the mechanical point of view, the solution can only contain one free parameter, so there must be a relation between $\rho_{0}$ and $c$ We consider the ratios of the shrinkage in thickness direction and in $R$ direction at $\Theta=0$ Since $\tau_{r r}=\tau_{r \theta}=\tau_{z z}=0$ at $\Theta=0$, it is required that $\frac{\partial r}{\partial R}=\frac{\partial z}{\partial Z}$ at $\Theta=0$, then according to $(36)$, we have

$$
(1+\beta) \rho_{0}=\varsigma(0)
$$

Since $u, v$ are constants as given in (57), $\varsigma$ is also a constant determined by the algebraic equation (46), Eq (59) and (46) give

$$
c=\sqrt{2}(1+\beta)^{\frac{-2 n}{2(n-1)}} \rho_{0}^{\frac{2-4 n}{2(n-1)}}
$$

therefore $\rho_{0}$ becomes the unique parameter for the expanding sector 


\section{Matching conditions}

The solution for the shrinking sector SH when $\Theta \rightarrow 0$ must match with the solution for the expanding sector EX when $\xi \rightarrow \infty$

We know that when $\zeta \rightarrow \infty$ Eq (55) has the asymptotic expression,

$$
\rho=c \rho_{0} \xi \quad \omega=\frac{1}{c \xi}
$$

From (36) and (62), we obtain

$$
r=R^{1+\beta \alpha} \rho_{0} c \Theta \quad \theta=\frac{\pi}{2}-\frac{R^{\alpha}}{c \Theta}
$$

In the shrınkıng sector, when $\Theta \rightarrow 0$, we have

$$
\varphi=C_{\varphi} \Theta
$$

in which $C_{\varphi}$ is an arbitrary coefficient that is permitted since (25) is homogeneous

Similarly, when $\Theta \rightarrow 0$, we have

$$
\psi=C_{\psi} \frac{1}{\Theta}
$$

where $C_{\psi}$ is an arbitrary constant

Using (9), (63) and (64) we have

$$
r=R^{1-\delta} C_{\varphi} \Theta, \quad \theta=\frac{\pi}{2}-R^{\gamma} C_{\psi} \frac{1}{\Theta}
$$

Comparing (62) - (65) we find that if

$$
\begin{array}{ll}
\delta=\alpha-\beta, & \alpha=\gamma \\
C_{\varphi}=c \rho_{0}, & C_{\psi}=\frac{1}{c}
\end{array}
$$

then functions $r, \theta$ are matched at the boundary between sectors EX and SH

From (66) and (17) we also find

$$
\beta=t=b
$$

Eq (68) shows that for sectors EX and $\mathrm{SH}$, the shrınkıng ratio in the thickness direction has the same order

Now, we check the stress singularitıes for both kınds of sectors From Eq (21) we know that the singularity of the dominant stress $\left(\tau_{r r}\right)$ in shrinking sector is

$$
\tau \sim R^{3 \delta-2 \gamma-2 n \delta}
$$

In expanding sector, the singularity of the stress is

$$
\tau \sim R^{\alpha-2 \beta-b-2 n(\alpha-\beta)}
$$

Comparing the order of (69) with (70) we find that the stresses have the same order in sector EX and $\mathrm{SH}$

\section{Conclusions}

\& The mode I crack tip in a rubber like material obeying the elastıc law of Gao (1997) under plane stress condition is composed of two shrinking sectors and one expanding sector When the crack tip is approached, the dominant stress possesses the singularity of order $R^{-\lambda}$, $\left(\lambda=1+\frac{2}{1+n}-\frac{3}{2 n}\right)$

$\diamond$ The thıckness shrınkage near the crack tıp is tremendous After deformation the thickness becomes the order of $R^{\frac{n-1}{2 n(n+1)}}$ both in expanding and shrinkıng sectors

$\diamond$ Although the elastic law used in this paper is different from that in Gao and Durban (1995), the crack tip is still in uniaxial tension state, but the equations for minor quantities are different

\& Both elastic law, Gao (1990) and Gao (1997) are valıd to analyze the crack tip in rubber sheet, but Gao (1997) is simpler than Gao (1990) 


\section{Acknowledgments}

This work is supported by the National Science Foundation of China, No 19572001 and No 19772001

\section{References}

Gao, Y C (1990), Elastostatic crack tip behavior for a rubber-like material, Theor Appl Fract Mech Vol 14, PP 219-231

Gao, Y C (1997), Large deformation field near a crack tıp in rubber-lıke materıal, Theor Appl Fract Mech Vol 26, PP 155-162

Gao, Y C and Gao, T (1999), Analytical solution to a notch tip field in rubber like materials under tension, Int J Solids Struct Vol 36, PP 5559-5571

Gao, Y C and Durban, D (1995), The crack tip field in a rubber sheet, Eur J Mech, A/Solids, Vol 14, PP 665-677

Knowles, J K and Sternberg, E (1973), An asymptotic finite deformation analysis of the elastostatic field near the tip of a crack, J Elasticity, Vol 3, PP 67-107

Knowles, J K and Sternberg, E (1974), Finite deformation analysis of the elastostatıc field near the tip of a crack reconsideration and higher-order results, J Elastıcity, Vol 4, PP 201-233 